\title{
PCR: paths to sensitivity
}

\author{
Vivien Marx
}

Careful strategizing helps make PCR sensitive enough for the most challenging experiments.

Scientists are rarely starved for choice when considering methods or technologies they could use to answer their next burning question or test a pet hypothesis. This is certainly the case for PCR. This workhorse technique underwent what some term a 'revolution' in the 1990s with the development of quantitative PCR (qPCR). Now the maturing of an alternative technique, digital PCR ( $\mathrm{dPCR}$ ), often leaves researchers wondering which is the right match for them.

For some, the method of choice may be qPCR, in which one or many molecules are subjected to repeated copying en masse and the amplification signal is interpreted. For others it may be the newer APCR technology, in which a sample is subjected to limiting dilution into many partitions or droplets. In such a highly diluted mixture, each volume has a reasonable chance of containing either one or zero molecules of the analyte before amplification. A reported signal is therefore likely to come from a single template molecule, yielding quantitative results. Some research groups even opt for both PCR methods to assure results are reproducible. Here, a few scientists share rationales for their choices in a variety of applications.

\section{Using genes as levers}

Tumor-infiltrating lymphocytes (TILs), which mediate the immune response, are good news: finding them in colon cancer or ovarian tumors means better odds of survival for patients. But quantifying TILs with flow cytometry is cumbersome and error prone, particularly for solid tumors from which single-cell suspensions need to be made. Jason Bielas and colleagues at Fred Hutchinson Cancer Center have developed QuanTILfy, a better way to count TILs ${ }^{1}$.

The team leverages genetic rearrangements in the loci coding for T cell-specific

surface receptors (TCRs) to identify and count these cells using dPCR. The approach delivers the number of TCR arrangements per genome in a tissue sample and helps to assess a tumor's heterogeneity.

The team believes that QuanTILfy is more readily standardized than immunohistochemistry and less pricey than highthroughput sequencing-based approaches. Although they could use qPCR, Bielas says that technique is "semi-quantitative at best." Data analysis requires standard curves for absolute quantification, which is a challenge when multiple primer and probe combinations need to be evaluated, as in $\mathrm{T}$-cell diversity assays. The group notes that this makes qPCR harder to get to a point at which results can readily be compared from lab to lab.

\section{Counting HER2}

Finding ways to avoid variability and to lower costs motivates Bruno Ping, who manages a molecular diagnostics lab at Royal Surrey County Hospital in Guildford, UK. He needs sensitive assays to help with clinical decision-making: for example, to check whether breast cancer tissue shows an amplification of the HER2 (ERBB2) gene, which guides the choice of treatment.

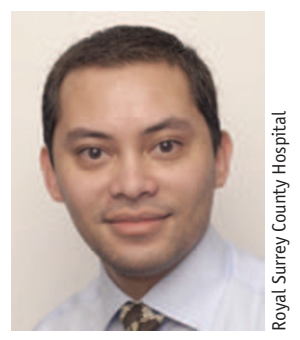

Using a normalizer gene when applying $d P C R$ to determine HER2 copy number is important, says Bruno Ping.
HER2-positive breast cancers can have extra copies of the HER 2 gene. They might have elevated levels of the HER2 protein, which is also called epidermal growth factor receptor 2 .

HER2 testing includes immunohistochemistry and in situ hybridization

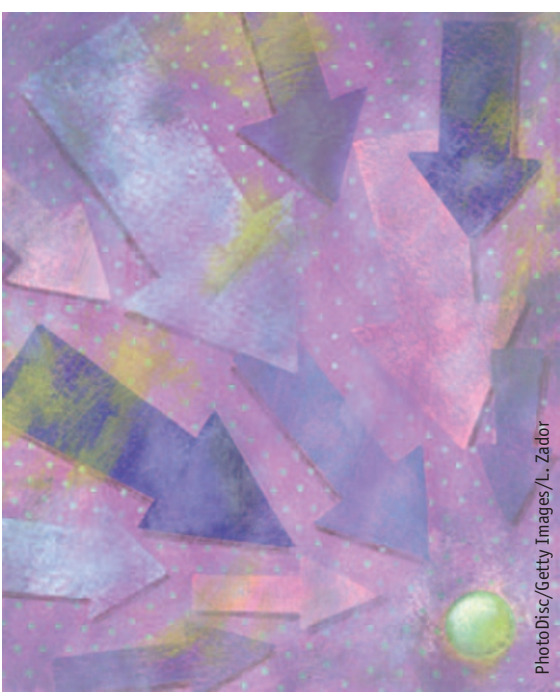

Detecting rare mutations or slight differences in gene expression with PCR is tricky.

for HER2. The classic method of fluorescence in situ hybridization (FISH) is reliable but challenging, Ping says. His group also introduced silver-enhanced in situ hybridization (SISH), which his colleagues in the histology department now use. But sometimes histological tests are inconclusive and need clarification. These cases, as well as cost factors, add to his team's motivation to explore new approaches.

Ping works on new research approaches to obtain sensitive, precise and accurate test results. After reading about qPCR and dPCR to assess HER 2 amplification, he tried dPCR because it delivers statistical results. For example, a sample might be $30 \%$ tumor, showing HER 2 amplified ten times. Making inferences from qPCR data about such a sample is difficult because, as Ping says, the data are "the sum of all tumor and nontumor content."

He has calculated that moving from SISH to dPCR on either the Bio-Rad or Life 
Technologies platform can mean between $10 \%$ and $90 \%$ in cost savings. The savings are even greater when shifting from FISH to dPCR.

These technologies are not mutually exclusive. "In the HER2 copy numbervariation case, we could use $\mathrm{dPCR}$ as a first pass and then reflex to SISH as a second pass for problem samples," says Ping. In that case, he found that this approach would cut down SISH testing by $70 \%$ and save upwards of $\$ 150,000$ a year.

Both qPCR and dPCR have benefits over slide-based methods, such as lower cost, time savings and less inter-observer variability. But culture plays a role, too. Some scientists and clinicians have a higher comfort level when looking at a slide versus a numerical value.

Using dPCR to analyze HER2 copynumber variation requires a cautious approach, including adherence to new guidelines issued by the American Society of Clinical Oncology and the College of American Pathologists. Ping appreciates that these guidelines focus more on absolute copy number.

Scientists can look at a cell under a microscope and count the HER2 signals inside the nucleus, repeat the process for 40 or 50 more cells, and come to an average count of absolute HER2 signals.

In qPCR this is hard to do, Ping says, because all the DNA from all the cells is mixed together, and the only way to determine whether there is an increase in HER2 copy number is to calculate a ratio. So when doing qPCR, one uses a gene whose copy number is expected not to increase, such as the $\beta$-globin gene, for normalization.

For dPCR, his lab applies a similar approach using a normalizer gene, although he notes that his copy number-variation work with HER2 on dPCR may not be the standard dPCR approach. A sample with 50 tumor cells will have a much lower absolute HER2 signal than a sample with 5,000 tumor cells. Without a normalizer gene, he says, it becomes difficult to reach a consensus on a threshold for samples that are positive for HER2.

\section{Mitochondrial DNA detective}

Scientists can be confounded by their findings. Experiments need to be repeated. When the results repeat, excitement can build. "The results are what lead us, and we have to listen to the results," says neu-

robiologist Ramon Trullàs, whose lab is at Instituto de Investigaciones Biomédicas de Barcelona, a research institute. He belongs to several consortia including one devoted to neurodegenerative diseases called Centro de Investigación Biomédica en Red de Enfermedades Neurodegenerativas (CIBERNED). This affiliation strategy gives his and similar labs precious access to resources funding and core facilities in Spain, where the science funding situation is dire.

Even under these financial constraints, he and colleagues want to contribute to the

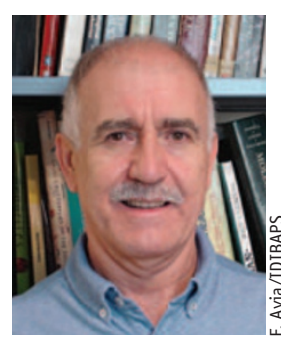

It was "extremely necessary" to use qPCR followed by $d P C R$, says Ramon Trullàs.

\section{Trullàs obtained}

cerebrospinal fluid samples from 282 patients, including a healthy group; a group with Alzheimer's; a group with frontotemporal lobar degeneration, which involves memory loss but also symptoms unlike those seen in Alzheimer's patients; and groups who are symptom free but at risk for the disease owing to low amyloid- $\beta$ levels in their cerebrospinal fluid or who carry a presenilin 1 (PSEN1) mutation, which is implicated in early onset Alzheimer's.

Using qPCR, because it was the technology most available to them, they measured 'cell-free' mitochondrial DNA (mtDNA) levels in cerebrospinal fluid. The circular molecule of double-stranded mtDNA leaves cells for reasons that are unclear. If neurons begin dying before the onset of Alzheimer's symptoms, the team would have expected to see large amounts of mtDNA in the fluid. The opposite was the case. In the samples from people with or at risk of developing Alzheimer's, the scientists found lower amounts of mtDNA. Trullàs, his postdoctoral fellow Petar Podlesniy and their colleagues spent five years troubleshooting this surprising result, which they have now published $^{2}$.

In live neurons, mitochondria shuttle through to the nerve terminal and then return to the neuronal cell body, where they fuse with other mitochondria. Watching time-lapse movies of this process is so beautiful, it gives him goose bumps, Trullàs says. But after seeing the qPCR results of cell-free mtDNA, there were no goose bumps. He recalls saying that "people won't believe uswe have to measure this with some other technique." They continued their imaging of mitochondria in intact neurons and tried additional techniques to study mtDNA inside and outside of neurons.

In cultured neurons from mouse models of Alzheimer's, they found a reduced number of mtDNA copies. The team redid the qPCR for cell-free mtDNA and tried experiments with antibodies, as well as an approach to image mtDNA with atomic force microscopy.

In 2011, the team learned about dPCR and set out to try it, but they knew of no lab with a dPCR instrument. Many phone calls later, Trullàs located a dPCR-equipped lab in Madrid, which was five hours away. The team decided to travel and run their samples there, where they confirmed their results: lower mtDNA levels are somehow correlated with the occurrence and risk of Alzheimer's. Trullàs is cautious about his findings. In response to his paper, groups from the US, Germany and The Netherlands have been sending Trullàs samples to test. $\mathrm{He}$ is also eager to see whether other groups can reproduce his findings. If the results are confirmed in other labs, mtDNA could help explain what contributes to Alzheimer'sthat neurons are losing their energyproducing ability.

With dPCR, he liked that there was no need to purify DNA from the cerebrospinal fluid, which contains many salts that inhibit

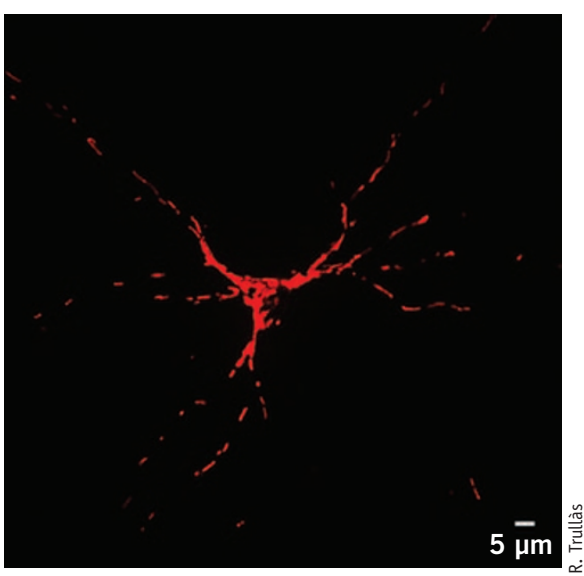

Mitochondrial DNA may offer hints about Alzheimer's disease. Here, a cultured live mouse neuron shows mitochondria labeled with the fluorescent marker DsRed. 
the $\mathrm{PCR}$ reaction. In $\mathrm{qPCR}$, obtaining accurate quantification means performing a continuous amplification curve without interference from inhibitors to the PCR reaction, Trullàs says. In $\mathrm{dPCR}$, the presence or absence of the PCR reaction is measured in a droplet. Even when there is some salt-based inhibition, the PCR reaction will still occur and quantify the molecules in an absolute sense. If, however, inhibition is so strong that the PCR reaction is utterly inhibited, there will be no positive droplets. This is where the importance of controlled experiments comes in, he says. He and his team checked and found that in their case, PCR inhibitors in cerebrospinal fluid did not influence dPCR but did have a strong influence on qPCR.

Quantitative PCR is sensitive in that it detects as little as a single DNA molecule, but findings can be hard to reproduce, Trullàs says, particularly when researchers try to detect low levels of DNA consistently. He felt it was "extremely necessary" in this case to do a well-optimized qPCR followed by dPCR, which is still a young technique. Once $\mathrm{dPCR}$ becomes more established and is easily accessible, scientists might not need to test with both methods. They might regularly do an assay with the most accessible technique for them, which may be qPCR, and then confirm a subset of samples with dPCR, he says.

Efforts are under way to comparatively assess the performance of $\mathrm{qPCR}$ and $\mathrm{dPCR}$. A recent study systematically compared the performance of qPCR with that of $\mathrm{dPCR}$ using droplets. The authors confirmed, using a variety of targets in different sample backgrounds, that dPCR with droplets showed greater precision and more reproducible findings ${ }^{3}$. The authors also speculate that it might be more "resilient" to sample quality differences. But, perhaps surprisingly, they failed to detect a significant difference in sensitivity under their controlled intralaboratory conditions.

Assays must certainly be optimized for the experimental conditions and instruments. Trullàs also recommends adhering to the Minimum Information for Publication of
Quantitative Real-Time PCR Experiments (MIQE) and digital MIQE guidelines and keeping vigilance levels high about factors that might contaminate samples.

\section{PCR school}

Scientists may think PCR is simple, says Ghent University researcher Jo Vandesompele, "but it's not." He splits his time between his own research and Biogazelle, a university spin-out that he cofounded and of which he is CEO. His team developed qPCR analysis software called

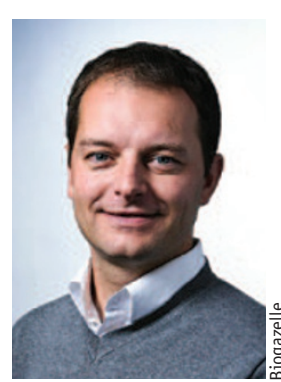

Scientists may think PCR is simple, says Jo Vandesompele, "but it's not." qbase+, and he has a statistical algorithm and software for dPCR in the works. He consults and sponsors training in PCR techniques to help a lab or a company department standardize its practices and to strategize about experiments. Courses have focused on qPCR best practices, and this year he is adding dPCR.

To those who say $\mathrm{dPCR}$ will replace the "very reliable" qPCR, he responds: "of course not." Digital PCR, however, might be right for some applications such as detection of rare mutations or copy-number variants.

He believes that qPCR is the "technology of choice" for gene expression-based experiments, particularly measuring gene expression changes of more than twofold, which probably make up the majority of experiments. In those instances, "qPCR can do the job" and at a lower price point that $\mathrm{dPCR}$, he says. But spotting gene expression differences among similar molecules such as splice variants or microRNAs might work better with dPCR.

Scientists using both qPCR and dPCR are bound to be happy about the price drop of dPCR instruments from Bio-Rad and Life Technologies, for example, in the last two years. And it might lead them to start their

\section{BOX 1 dPCR TIPS}

- Heed the guidelines: MIQE and its sibling, digital MIQE

- The efficiency of the PCR reaction still matters

- PCR inhibitors can skew both qPCR and dPCR results

- There's a need for statistical analysis methods

Source: J. Vandesompele, Ghent University/Biogazelle 


\section{BOX 2 ASSAYS TO GO}

A number of companies offer qPCR and dPCR assays, including Life Technologies, Qiagen and EMD Millipore. Bio-Rad has been expanding its assay series called PrimePCR, which are wet lab-validated, MIQE-compliant assays with probes, primers and documentation for qPCR and dPCR, says Bio-Rad's Sam Ropp, who runs the company's division of genomics reagents.

For qPCR, Bio-Rad offers validated gene expression assays for human and mouse. Rat genome assays are in the works through a collaboration with Biogazelle and Integrated DNA Technologies.

Given the information in a validated report, including sequencing information, researchers can see what their experiment is detecting, Ropp says. In gene expression experiments, there can be splice variants. There can be "false copies" from pseudogenes, which are similar in sequence to the genes scientists seek to detect. "Your primers will actually sit down on those targets and amplify them as well," he says of pseudogenes. In the worst case, a researcher will not know for sure what an experiment is detecting.

Typically, a scientist might take a gene sequence and feed it into a software package, and "it will pop out primers," says Ropp. But not all software tools are equal: for example, in the way they show regions of sequence homology, which scientists want to avoid.

Ignoring the assay in both qPCR and dPCR can render irrelevant "how awesome the rest of the technology is," he says. Even if scientists make their own probes and primers, such tools become trustworthy only with wet-lab validation. When scientists in small labs or core facilities or labs certified to test patient samples design their own assays for rare-mutation detection, the design iteration can be challenging because there might be only a single-nucleotide mismatch between the reference sequence and the one to be detected, says Bio-Rad's George Karlin-Neumann.

Reagents can also perform differently even under slightly different experimental conditions. For dPCR, the company assays are validated on Bio-Rad's Droplet Digital PCR system, and the company offers rare-mutation detection and copy numbervariation probe assays with various fluorophore chemistries.

As Jo Vandesompele of Ghent University and Biogazelle says, a suboptimal assay will reflect poorly on qPCR and $\mathrm{dPCR}$ results. When validating assays, he and his team look at the way primers and probes behave relative to the mix of enzymes and buffers for a specific instrument.

Biogazelle took on the design and wet-lab validation of qPCR and a subset of dPCR assays for Bio-Rad. Vandesompele's team has validated around 80,000 assays in the last 18 months, with Integrated DNA Technologies synthesizing the oligonucleotides. "They're one of the only companies that can do it at that scale, that turnaround time and that quality," he says of Integrated DNA Technologies. And the validation report for the assays also helps scientists to have the information on hand to accompany their data in submitted papers, he says.

analysis directly with dPCR, he says.

Sensitivity is one word Vandesompele avoids in a PCR context because both qPCR and APCR are sensitive enough to detect one molecule. But dPCR has a "far superior relative sensitivity," he says, enabling quantification of a single molecule against a background of many others that are similar, with higher accuracy and precision.

For example, a researcher might be looking for a cancer allele in a majority background of wild-type alleles. In qPCR, the majority alleles compete with the minority species, but in dPCR the majority species are diluted out. "You can do a much better job in detecting and quantifying these minority variants," Vandesompele says.

He says the large dPCR instrument manufacturers have chosen 20,000 droplets or partitions for PCR reactions, but others such as RainDance choose millions of droplets. For detecting one mutant cancer cell in a background of 1 million normal cells, those millions of droplets are superior; but, he says, for the majority of applications, 20,000 reactions is a technology "sweet spot."

Although dPCR instruments use proprietary algorithms to deliver results, they go from droplets to the absolute values almost equally well. Scientists know qPCR instruments have many settings and downstream processing details to heed. Although dPCR is relatively simple in comparison, he sees some emerging dPCR misconceptions (Box 1).

Most, but not all, dPCR instruments use Poisson-based statistics. Vandesompele sees room for methods development related to confidence calculations, to assure scientists of their results. Combining results of PCR reactions in droplets or partitions is "more tricky," he says, because each one carries its own uncertainty. Mathematical models for that purpose are lacking, which is why he and his colleagues are working on a new statistical approach based on general linear mixed modeling.

The next few years will see much dPCR methods development, just as the late 1990s did for qPCR. More applications will also emerge as scientists see the "powers" of dPCR. But qPCR and dPCR will remain complementary technologies, he says.

\section{Market views}

Instrument manufacturers interviewed by Nature Methods say that most dPCR instruments are delivering output of similar quality. They also agree that many dPCR applications are just emerging. But they note differences between offerings. For example, instruments differ in the number of partitions or droplets that can be interrogated.

As a type of 'single-molecule counter', the RainDrop dPCR system can determine molecule identity from the ratio of its two fluorescent signals, which moves measurement beyond the more typical single-colorper-marker approach, says Rena McClory, who directs marketing at RainDance Technologies.

The sensitive measurements are due to the instruments' many picoliter-sized droplets, each containing a single target molecule. The platform handles up to ten million reactions per sample and lets scientists multiplex up to ten targets per sample.

Many customers run tests on both qPCR and $\mathrm{APCR}$ platforms to validate their results, but McClory anticipates that most of them will switch to dPCR completely, especially in medical genetics and clinical labs. She sees an edge for her company's platform in its ability to detect low-frequency alleles, copynumber variation and small differences in gene expression, as well as for its usefulness in viral detection. 

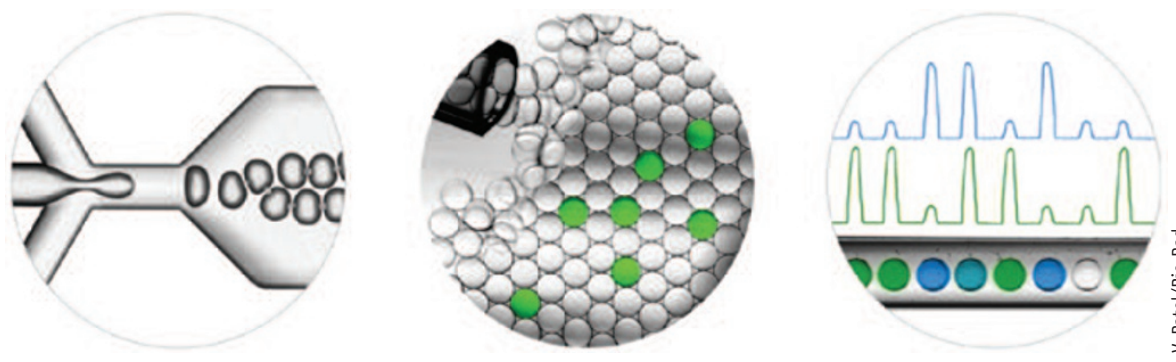

In Bio-Rad's dPCR workflow, reaction mixes are partitioned into droplets (left). The PCR reaction takes place in each droplet (center). If the target sequence is amplified, a reporter dye emits a fluorescent signal, which is read (right).

Gene-expression researchers probably have different answers to the question "Would you like to be more sensitive?" says Bio-Rad's Sam Ropp, whose company sells both qPCR and dPCR platforms. Some might use the term 'more sensitive' to describe a twofold change. For others, it relates to monitoring a gene that is rarely expressed. Most gene expression experiments look at changes such as the difference between 10,000 and 20,000 copies, which qPCR can handle well, he says. A caveat for both qPCR and dPCR, says Ropp, is

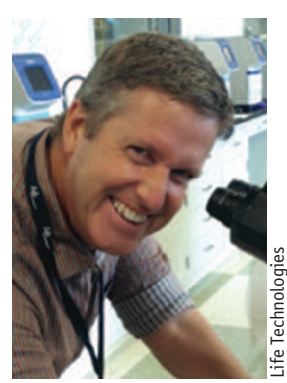

Partitions on a chip avoid potential clogging in microfluidics-based systems, says Stephen Jackson. that the assays may

not be monitoring what a researcher thinks they are (Box 2).

When it comes to copy-number variation, in Ropp's view, most dPCR instruments can detect a single copy or 10 or 100 . Whether the reactions are spread over 20,000 droplets or 1 million does not matter as much as the experimental protocols, reagents, assays and data analyses.

The right combination of instrument, assays and analysis helps researchers to find potential biomarkers in the bloodstream for cancer or infectious disease or to detect harmful bacteria in the water supply, says George Karlin-Neumann, who directs scientific affairs at Bio-Rad.

Last year, Life Technologies began instrument, in which 20,000 PCR reactions take place in small partitions on a chip. The partitions avoid potential clogging in microfluidicsbased systems, says Stephen Jackson, who directs research in the genetic analysis division at Life Technologies.

Scoring reaction results occurring in the partitions is a more accurate and precise measurement than qPCR output of what is in a sample, he says. Because there can be more than one gene copy or molecule in the positive partitions, in Life Technologies' platform, the software counts the negatives and not the positives. A calculation using a Poisson distribution delivers to the user the number of molecules per partition and in the sample.

In addition to the direct instrument readout, Life Technologies offers QuantStudio 3D data analysis software. Researchers can analyze and view data across replicates, plates and experiments and can choose between server-based and cloud-based options.

Jackson agrees with others that dPCR's strength is its ability to discriminate selling its QuantStudio 3D dPCR between, for example, four and five gene copies. The numbers can indicate disease severity, which is one reason why researchers explore dPCR for copy numbervariation analysis.

Digital PCR has researchers thinking of many potential applications in medical diagnostics, Jackson says. One day, the instrument might not be just in labs and core facilities but in doctor's offices, too. It has been engineered so that it can be used by nonspecialists, he says.

Digital results are another advantage of $\mathrm{dPCR}$, which is better than interpreting fluorescent dye brightness in a qPCR array. Looking for rare mutations that identify a diseased cell in a sea of DNA from normal cells is a task well suited to dPCR, he says.

Millions of partitions or droplets can be too many in a clinical application, says Jackson. Life Technologies' platform uses 20,000 partitions, which means around 50-60 nanograms of genomic clinical sample are needed. More partitions would mean micrograms of sample. Extra partitions might help in other applications: for example, when scientists analyze a wheat field and 60-microgram samples are more readily available.

Despite all of its advantages, Jackson says dPCR is "not going to be the hammer that fits every nail.” Plenty of applications remain for which $\mathrm{qPCR}$ is the apt choice.

Difficult challenges that call for sensitive, precise and accurate measurement, mean that decision-making approaches, too, need to be sensitive, precise and accurate.

1. Robins, H.S. et al. Sci. Transl. Med. 5, $214 \mathrm{ra} 169$ (2013).

2. Podlesniy, P. et al. Ann. Neurol. 74, 655-668 (2013).

3. Hindson, C.M. et al. Nat. Methods 10, 10031005 (2013).

Vivien Marx is technology editor for Nature and Nature Methods (v.marx@us.nature.com). 